\title{
Gingivitis descamativa asociada a penfigoide de membranas mucosas
}

\author{
Israel Guardado Luévanos, ${ }^{*}$ Patricia Guzmán Beltrán, ${ }^{\ddagger}$ Mario Nava Villalba, ${ }^{*}$ \\ Miguel Padilla Rosas, ${ }^{*}$ Sandra López Verdín, ${ }^{\S}$ Julio Miguel Peña Ruelas"l
}

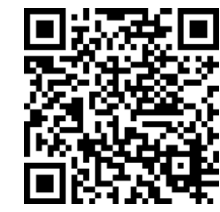

\section{RESUMEN}

La gingivitis descamativa asociada con penfigoide de membranas mucosas (MMP) (ICD-10 L10), es una entidad correspondiente a enfermedad gingival no relacionada con placa bacteriana, que forma parte de un grupo heterogéneo de desórdenes ampollares, autoinmunes y crónicos, que se manifiestan clínicamente como áreas con eritema sintomáticas sobre la encía, las cuales forman una bulla ante el frotamiento, lo que se conoce como Nikolsky positivo. El estudio histopatológico se caracteriza por la división sub-basal, con presencia de infiltrado inflamatorio en la lámina propia pertinente a los depósitos de proteínas del complemento e inmunoglobulinas. El examen de inmunofluorescencia directa muestra depósitos lineales de inmunoglobulinas, principalmente IgG y complemento sobre la membrana basal. Se describe un caso donde se examinó la relación entre el periodoncista y el patólogo bucal, para el diagnóstico de penfigoide de membranas mucosas.

Palabras clave: Penfigoide de membranas mucosas, gingivitis descamativa, inmunofluorescencia.

\section{INTRODUCCIÓN}

Prinz propuso el concepto de «gingivitis descamativa» (DG), en 1932, para indicar la presencia de eritema, descamación, erosión y ampollas, en la mucosa oral marginal y adherida, ${ }^{1}$ signos que representan diferentes trastornos mucocutáneos como: dermatosis, factores hormonales, irritantes e idiopáticos. Las dermatosis incluyen penfigoide de la membrana mucosa, pénfigo y liquen plano. ${ }^{2}$

* Maestría en Patología y Medicina Bucal, Centro Universitario de Ciencias de la Salud, Universidad de Guadalajara.

‡ Especialista en Patología y Medicina Bucal, Universidad Autónoma Metropolitana Xochimilco.

$\S$ Instituto de Investigación en Odontología, Centro Universitario de Ciencias de la Salud, Universidad de Guadalajara.

\| Especialidad en Periodoncia, Centro Universitario de Ciencias de la Salud, Universidad de Guadalajara.

Recibido: 10 de octubre de 2019. Aceptado: 04 de diciembre de 2019.

Este artículo puede ser consultado en versión completa en www.medigraphic.com/periodontologia

\begin{abstract}
Desquamative gingivitis associated with penfigoid mucous membranes (MMP) (ICD-10 L10), is an entity corresponding to gingival disease not related to bacterial plaque that is part of a heterogeneous group of ampullary, autoimmune and chronic disorders, which manifest clinically as areas with symptomatic erythema on the gum, that when rubbing form a bulla, which is known as Nikolsky positive. The histopathological study is characterized by sub-basal division with the presence of inflammatory infiltrate in the lamina propria relevant to the deposits of complement proteins and immunoglobulins. The direct immunofluorescence test shows linear deposits of inmonuglobulins, mainly IgG and complement on the basement membrane. We describe a case examined the relationship between the periodontist and the oral pathologist for the diagnosis of mucous membrane pemphigoid.
\end{abstract}

Keywords: Pemphigoid of mucous membranes, desquamative gingivitis, immunofluorescence.

El penfigoide de la membrana mucosa corresponde a un grupo heterogéneo de trastornos ampollosos autoinmunes, caracterizados por la separación subepitelial y el depósito de inmunoglobulinas y complemento a lo largo de la zona de la membrana basal. ${ }^{3}$ Presenta un amplio espectro de gravedad de la enfermedad y afectación del sitio clínico que incluye lesiones orales, oculares, dérmicas, nasofaríngeas, esofágicas, entre otras. ${ }^{4-9}$

Debido al curso clínico del penfigoide de la membrana mucosa, es necesario un diagnóstico temprano y un tratamiento adecuado. El diagnóstico de penfigoide de las membranas mucosas se basa en al menos cuatro criterios: 1) presencia de una vesícula o proceso ampolloso que involucra las membranas mucosas; 2) histología de la lesión, que muestra la presencia de una ampolla subepitelial con un infiltrado inflamatorio compuesto de neutrófilos, entre otras células inflamatorias; 3) presencia de deposición lineal continua de anticuerpos $\operatorname{IgG}$, IgA y/o C3 específicos a lo largo de la membrana basal en el estudio de inmunofluorescencia directa (IFD) 
de biopsia perilesional; y 4) detección de autoanticuerpos IgG e IgA contra la membrana basal en el suero, mediante pruebas de inmunofluorescencia indirecta (IIF). ${ }^{10-13}$

Los dentistas son los proveedores de atención clave para establecer diagnósticos y formular planes de tratamiento para pacientes afectados por tales lesiones. Los especialistas en periodontología deben estar familiarizados y ser capaces de diagnosticar, tratar o derivar para el tratamiento de cualquier lesión de este tipo. ${ }^{14}$

Presentamos un caso de gingivitis descamativa asociada con penfigoide de las membranas mucosas, que requirió el manejo por el área de periodoncia y patología bucal para el diagnóstico y tratamiento, y así obtener el control de las lesiones de la mucosa.

\section{Descripción DEL CASO CLÍNICO}

Masculino de 61 años, quien acudió a consulta a la clínica de maestría en patología y medicina bucal CUCS UdeG, derivado por la Clínica de Especialidad de Periodoncia; sufría ardor y malestar en boca, con un tiempo de evolución de tres años. Diabético bajo tratamiento con metformina e hipertenso tratado con benazepril y lovastatina.

El paciente informó que su dentista de atención primaria realizó un examen de biopsia previo con diagnóstico histopatológico de proceso inflamatorio crónico inespecífico. Se indican pruebas de laboratorio que incluyeron biometría hemática, tiempo de protombina y triglicéridos. Estas pruebas fueron esencialmente normales, con la excepción de los triglicéridos, que estaban elevados.

El examen oral reveló áreas erosivas y sangrado significativo al palpar los tejidos gingivales. Las lesiones se limitaron a la encía adherida maxilar y mandibular con un patrón descamativo clásico y presencia leve de placa dentobacteriana (Figuras $1 A$ y $1 B$ ). Se percibe pérdida de inserción clínica; sin embargo, no se registran valores significativos al sondeo periodontal.

Se realizó una biopsia gingival y se sometieron a un examen histológico e inmunofluorescencia directa. La histología demostró un espécimen cubierto por un epitelio estratificado paraqueratinizado, con ligera acantosis y extensas zonas con pérdida de solución de continuidad (Figura 2).

Con base en los hallazgos clínicos, histológicos, incluida la presencia de anticuerpos lineales sobre la

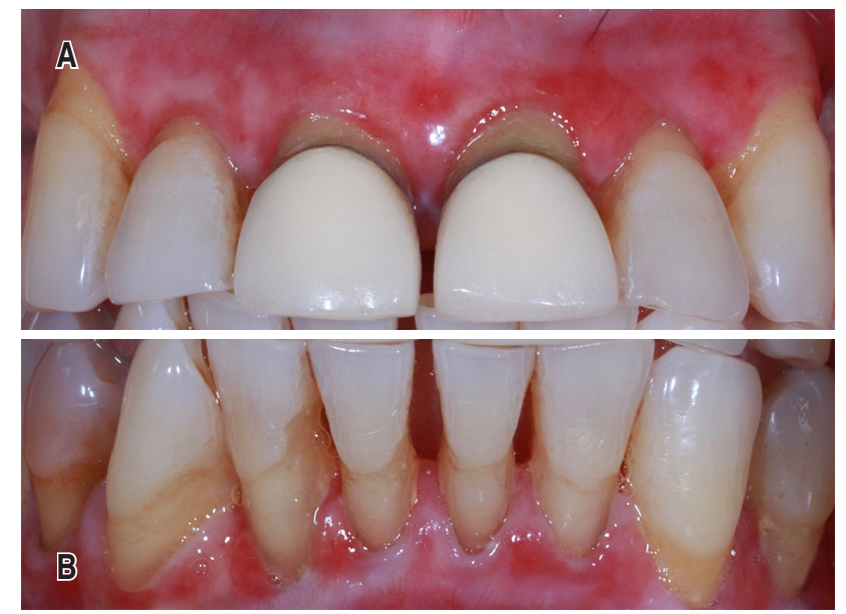

Figura 1: A) Imagen de fotografía frontal correspondiente al sextante anterior superior, en el que se observa eritema y ulceración en encía adherida. B) Imagen de fotografía del sextante anterior inferior, nótese la cantidad de placa bacteriana, la cual no está asociada con los signos de inflamación sobre la encía adherida.

membrana basal IgG y C3 en la inmunofluorescencia directa (Figura 3), se estableció un diagnóstico de penfigoide de las membranas mucosas que se presenta con gingivitis descamativa.

Se indica tratamiento con corticoesteroide (clobetasol) y antimicótico (nistatina); así como eliminación de placa supragingival y refuerzo de control de placa personal. Se mantiene en observación mediante la valoración periodontal, con atención al control de placa bacteriana, así como evaluación por parte del patólogo bucal de la evolución de las lesiones gingivales autoinmunes.

\section{Discusión}

La gingivitis descamativa representa un trastorno de la mucosa oral relevante, debido a la diversidad de su origen. Por esto, no siempre es posible relacionar la DG con un diagnóstico previo, es importante que los especialistas en salud oral conozcan su concepto para poder distinguir entre una gingivitis inflamatoria clásica y una DG asociada con la placa dental. ${ }^{15}$

El médico debe identificar no sólo la descripción clínica de su presencia de eritema, erosión y/o ampollas, sino que también debe hacer un estudio microscópico convencional e inmunofluorescencia. Este hecho requiere que el médico especialista, 
generalmente en patología oral, realice la biopsia en el área apropiada, con el propósito de proyectar el diagnóstico histopatológico e inmunológico de la lesión. ${ }^{16}$

El penfigoide de la membrana mucosa es una enfermedad autoinmune rara, crónica, que suele presentar gingivitis descamativa, en la cual los autoanticuerpos, predominantemente BP180, se forman contra los componentes de la membrana basal. Afecta comúnmente a mujeres de mediana edad y ancianas. La presentación clínica consta de vesículas/ampollas en la mucosa oral, que involucra la encía. Suele manifestarse como un eritema doloroso con descamación, ya sea espontáneo o después de un traumatismo menor, como el cepillado de dientes. ${ }^{17}$

Diversos estudios reportan terapias específicas que se han centrado en las manifestaciones generales de la enfermedad, relacionadas con su patogénesis. Se ha indicado el tratamiento tópico, principalmente el uso de corticosteroides en diferentes formas y prescritos con diferentes posologías, o también la administración de fármacos sistémicos como corticosteroides, otros inmunosupresores y antibióticos de amplio espectro. ${ }^{18,19}$ Sin embargo, el estado periodontal en pacientes con gingivitis descamativa asociada con penfigoide de membranas mucosas es peor, ${ }^{20-22}$ presentan bolsas más profundas y una mayor pérdida del nivel de inserción clínica. ${ }^{23,24}$

Estudios presentados respaldan la eficacia de mantener la higiene oral en pacientes con DG asociada con penfigoide de membranas mucosas, reduciendo los signos clínicos de la enfermedad.

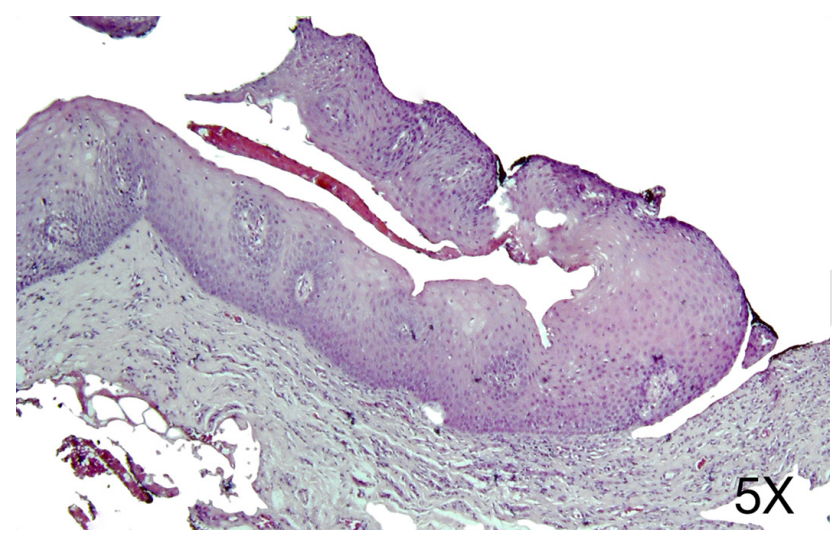

Figura 2: Microfotografía que muestra la pérdida de solución de continuidad. Se observa una imagen panorámica (5x) del espécimen estudiado.

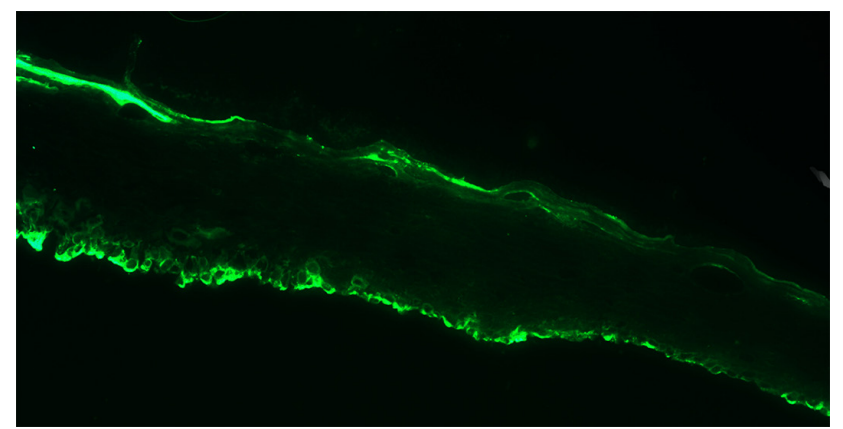

Figura 3: Microfotografía de estudio de inmunofluorescencia que muestra los depósitos de inmunoglobulina $(\lg G)$ sobre la membrana basal.

Los pacientes con DG deben recibir instrucciones por parte del periodoncista sobre el mantenimiento de una higiene adecuada, utilizando diferentes técnicas, cepillos de dientes con cerdas suaves o extra suaves, que se puedan insertar fácilmente sobre el surco gingival y el uso de hilo dental. ${ }^{25}$

\section{Conclusión}

Las enfermedades gingivales no relacionadas con placa bacteriana son menos comunes, pero de mayor significancia para el paciente, ya que manifiestan condiciones sistémicas y representan entidades con diagnósticos más allá de lo clínico. Por lo que la atención interdisciplinaria periodoncia-patología es necesaria para el diagnóstico preciso y tratamiento, con el propósito de erradicar condiciones inflamatorias (placa dentobacteriana), que podrían favorecer el desarrollo de las lesiones ulcerativas de manifestación sistémica, como el penfigoide de membranas mucosas.

\section{Referencias}

1. Sorrin S. Chronic desquamative gingivitis. JADA. 1940; 27: 250-257.

2. McCarthy PL, Shklar G. Disease of the oral mucosa: diagnosis, management and therapy. New York: McGraw Book Co.; 1964. pp. 183-191.

3. Hardy KM, Perry HO, Pingree GC, Kirby TJ. Benign mucous membrane pemphigoid. Arch Dermatol. 1971; 104: 467-475.

4. Person JR, Rogers RS III. Bullous and cicatricial pemphigoid: clinical, histopathological, and immunopathologic correlations. Mayo Clin Proc. 1977; 52: 54-66.

5. Rogers RS III, Perry HO, Bean SF, Jordon RE. Immunopathology of cicatricial pemphigoid: Studies of complement deposition. J Invest Dermatol. 1977; 68: 39-43. 
6. Rogers RS III, Sheridan PJ, Nightingale SH. Desquamative gingivitis: Clinical, histopathologic, immunopathologic, and therapeutic observations. J Am Acad Dermatol. 1982; 7: 729735.

7. Silverman S, Gorsky M, Lozada-Nur F, Liu A. Oral mucous membrane pemphigoid: a study of 65 patients. Oral Surg Oral Med Oral Pathol. 1986; 61: 233-237.

8. Foster C. Cicatricial pemphigoid. Trans Am Ophthalmol Soc. 1986; 84: 527-663.

9. Hanson RD, Olson KD, Rogers RS III. Upper aerodigestive tract manifestations of cicatricial pemphigoid. Ann Otol Rhinol Laryngol. 1988; 97: 493-499.

10. Chan LS. Mucous membrane pemphigoid. Clin Dermatol. 2001; 19: 703-711.

11. Chan LS, Ahmed AR, Anhalt GJ et al. The first international consensus on mucous membrane pemphigoid: definition, diagnostic, pathogenic factors, medical treatment, and prognostic indicators. Arch Dermatol. 2002; 138: 370-379.

12. Setterfield J, Shirlaw PJ, Kerr-Muir M et al. Mucous membrane pemphigoid: A dual circulating antibody response with IgG and IgA signifies a more severe and persistent disease. Br J Dermatol. 1998; 138: 602-610.

13. Venning VA, Allen J, Millard PR, Wojnarowska F. The localization of the bullous pemphigoid and cicatricial pemphigoid antigens: direct and indirect immunofluorescence of suction blisters. Br J Dermatol. 1989; 120: 305-315.

14. Holmstump P, Plemons J, Meyle J. Non-plaque-induced gingival diseases. J Clin Periodontol. 2018; 45 (Suppl 20): S28-S43.

15. Hassona Y, Cirillo N, Taimeh D, Al Khawaldeh H, Sawair F. Diagnostic patterns and delays in autoimmune blistering diseases of the mouth: a cross-sectional study. Oral Dis. 2018; 24: 802-808.

16. Maderal AD, Lee Salisbury P 3rd, Jorizzo JL. Desquamative gingivitis: clinical findings and diseases. J Am Acad Dermatol. 2018; 78: 839-848.
17. Sciubba JJ. Autoimmune oral mucosal diseases: clinical, etiological, diagnostic, and treatment considerations. Dent Clin N Am. 2011; 55: 89-103.

18. Maderal AD, Lee Salisbury P 3rd, Jorizzo JL. Desquamative gingivitis: diagnosis and treatment. J Am Acad Dermatol. 2018; 78: 851-861.

19. Karagoz G, Bektas-Kayhan K, Unur M. Desquamative gingivitis: A review. J Istanb Univ Fac Dent. 2016; 50: 54-60.

20. Arduino PG, Farci V, D’Aiuto F, Carcieri P, Carbone M, Tanteri $\mathrm{C}$ et al. Periodontal status in oral mucous membrane pemphigoid: initial results of a case-control study. Oral Dis. 2011; 17: 90-94.

21. Tricamo MB, Rees TD, Hallmon WW, Wright JM, Cueva MA, Plemons JM. Periodontal status in patients with gingival mucous membrane pemphigoid. J Periodontol. 2006; 77: 398-405.

22. Lo Russo L, Guiglia R, Pizzo G, Fierro G, Ciavarella D, Lo Muzio L et al. Effect of desquamative gingivitis on periodontal status: a pilot study. Oral Dis. 2010; 16: 102-107.

23. Azizi A, Rezaee M. Comparison of periodontal status in gingival oral lichen planus patients and healthy subjects. Dermatol Res Pract. 2012; 2012: 561232.

24. Thorat MS, Raju A, Pradeep AR. Pemphigus vulgaris: effects on periodontal health. J Oral Sci. 2010; 52: 449-454.

25. Garcia-Pola MJ, Rodriguez-López S, Fernánz-Vigil A, Bagán L, Garcia-Martín JM. Oral hygiene instructions and professional control as part of the treatment of desquamative gingivitis. Systematic review. Med Oral Patol Oral Cir Bucal. 2019; 24 (2): e136-e144.

Correspondencia:

Israel Guardado Luévanos

Av. López Mateos Núm. 567, Int. 207,

Col. Ladrón de Guevara, 44600,

Guadalajara, Jalisco, México.

Tel: (33) 33-1477-9335

E-mail: iguardadol@hotmail.com 